\section{Hiperparativeoidismo Primário em Paciente Com Lúpus Eritematoso Sistêmico}

\section{RESUMO}

O hiperparatireoidismo primário (HPP) é uma doença metabólica que resulta da secreção anormal do hormônio da paratireóide (PTH), sendo uma das causas mais comuns de hipercalcemia na população geral. Apresentamos o caso de uma paciente de 47 anos, com diagnóstico prévio de lúpus eritematoso sistêmico (LES) há dois anos, que apresentou manifestações clínicas (dor óssea difusa, labilidade emocional, tumoração em ramo mandibular esquerdo) e laboratoriais (cálcio séri$\mathrm{CO}=13,5 \mathrm{mg} / \mathrm{dL}$, fosfato $=1,8 \mathrm{mg} / \mathrm{dL}$, fosfatase alcalina $=3.028 \mathrm{U} / \mathrm{L}$, PTH intacto $=1472 \mathrm{pg} / \mathrm{dL}$ ) que culminaram com o diagnóstico de HPP, complementado pelo estudo anátomo-patológico, que evidenciou adenoma de paratireóide. Após o tratamento com calcitonina nasal 400Ul/dia, pamidronato $90 \mathrm{mg} \mathrm{IV} / \mathrm{semana}$ e, por fim, a paratireoidectomia subtotal, a paciente apresentou melhora do quadro clínico e normalização dos exames laboratoriais. A presente descrição se constitui no segundo caso relatado na literatura, com tal associação de doenças. Embora os mecanismos etiopatogênicos do HPP em LES não estejam ainda definidos, essa associação deve ser sempre lembrada, uma vez que a estratégia terapêutica tende a mudar completamente. (Arq Bras Endocrinol Metab 2004;48/4:555-558)

Descritores: Lúpus eritematoso sistêmico; Hiperparatireoidismo; Hipercalcemia; PTH

\begin{abstract}
Primary Hyperparathyroidism in a Patient With Systemic Lupus Erythematosus.

Primary hyperparathyroidism (PHP) is a metabolic illness that results from autonomous secretion of parathyroid hormone and is one of the most common causes of hypercalcemia. We present the case of a 47-yearold female with a previous diagnosis of systemic lupus erythematosus (SLE) in whom clinical (diffuse bone pain, emotional lability, jaw tumor) and laboratory features (calcium $=13.5 \mathrm{mg} / \mathrm{dL}$, phosphate $=1.8 \mathrm{mg} / \mathrm{dL}$, alkaline phosphatase $=3028 \mathrm{U} / \mathrm{L}$, PTH intact $=1472 \mathrm{pg} / \mathrm{dL}$ ) prompted the diagnosis of PHP secondary to parathyroid adenoma as demonstrated by the anatomopathology. After treatment with calcitonin spray $400 \mathrm{UI}$ per day, IV pamidronate $90 \mathrm{mg} /$ week, and subtotal parathyroidectomy, the patient status improved with normal laboratory tests. This is the second report to describe the coexistence of these two disorders in a single patient. Although the pathophysiology of the association of PHP and SLE is not known, the recognition of this association has a practical implication since the therapeutical strategy is completely different. (Arq Bras Endocrinol Metab 2004;48/4:555-558)
\end{abstract}

Keywords: Systemic lupus erythematosus; Primary hyperparathyroidism; Hypercalcemia; PTH apresentação de caso

\author{
Liliana D. Galvão \\ Isabella V. Lima \\ Luiza Santos \\ Mittermayer B. Santiago
}

Núcleo de Reumatologia da Bahia, Hospital Santa Izabel, Salvador, BA.

Recebido em 13/11/03

Revisado em 09/02/04 e 13/05/04

Aceito em 23/06/04 
$\mathbf{O}$ hiperParatireoidismo pRIMÁrio é uma doença metabólica que resulta da secreção anormal de paratormônio (PTH), sendo uma das causas mais comuns de hipercalcemia na população geral (1). Pode se apresentar clinicamente de diversas maneiras, variando da forma assintomática à hipercalcemia sintomática, doença óssea, litíase renal e crise paratireoídea (2). As manifestações reumatológicas observadas no hiperparatireoidismo são a condrocalcinose, dor óssea difusa, pseudogota e gota (3-5). Raramente pacientes com lúpus eritematoso sistêmico (LES) podem apresentar hipercalcemia. Essa poderia ser decorrente da presença de anticorpos estimuladores contra receptores de PTH (6), produção de peptídeo similar ao PTH (PTH-rP), mesmo na ausência de neoplasia (7), ou fazendo parte da chamada síndrome hipercalcemia/linfedema, que inclui adenomegalia e pleurite $(8,9)$. Em uma ampla revisão de literatura, encontramos um único relato de caso de associação de hiperparatireoidismo primário com lúpus eritematoso sistêmico (10). O objetivo do presente trabalho é apresentar o segundo caso da literatura e discutir a interface dessas duas condições.

\section{RELATO DO CASO}

J.A.M, sexo feminino, 47 anos, teve diagnóstico de LES há cerca de dois anos (artralgia, leucopenia, FAN, por imunofluorescência em HEp-2, positivo com padrão pontilhado fino, título $1: 1280$, anti-RNP $1: 400$, antiSSA 1:12.800), sendo acompanhada em outro serviço e vindo em uso de prednisona $5 \mathrm{mg} /$ dia. $\mathrm{Na}$ admissão, referia que há seis meses vinha cursando com astenia, cefaléia, dor óssea, labilidade emocional, perda ponderal e obstipação intestinal. Foi internada para esclarecimento diagnóstico. Ao exame físico, apresentava-se emagrecida, hipocorada, ansiosa, com tumoração em ramo mandibular esquerdo, sem alterações à palpação da tireóide. Exibia, também, acentuada cifoescoliose. Apresentava os seguintes exames na internação: cálcio sérico: $13,5 \mathrm{mg} / \mathrm{dL} \quad(8,5-10,5 \mathrm{mg} / \mathrm{dL})$; cálcio urinário: $336 \mathrm{mg} / 24 \mathrm{~h} \quad(<180 \mathrm{mg} / 24 \mathrm{~h})$; fósforo sérico: $1,8 \mathrm{mg} / \mathrm{dL}(2,5-4,5 \mathrm{mg} / \mathrm{dL})$; fosfatase alcalina $(\mathrm{FA})$ : $3028 \mathrm{U} / \mathrm{L}(50-250 \mathrm{U} / \mathrm{L}) ; \mathrm{PTH}$ intacto: $1472 \mathrm{pg} / \mathrm{mL}$ $(10-60 \mathrm{pg} / \mathrm{mL})$; uréia: $34 \mathrm{mg} / \mathrm{dL}$; creatinina: $1,0 \mathrm{mg} / \mathrm{dL}$. Raio-x de crânio: aspecto granular dos ossos da calota craniana com lesão em "sal e pimenta"; TC de mandíbula e tórax: lesões osteolíticas insuflantes, multilobulares compatíveis com "tumores marrons" (figura 1). Cintilografia de paratireóides com Sestamibi-Tc 99m: área de retenção tardia anormal do traçador na

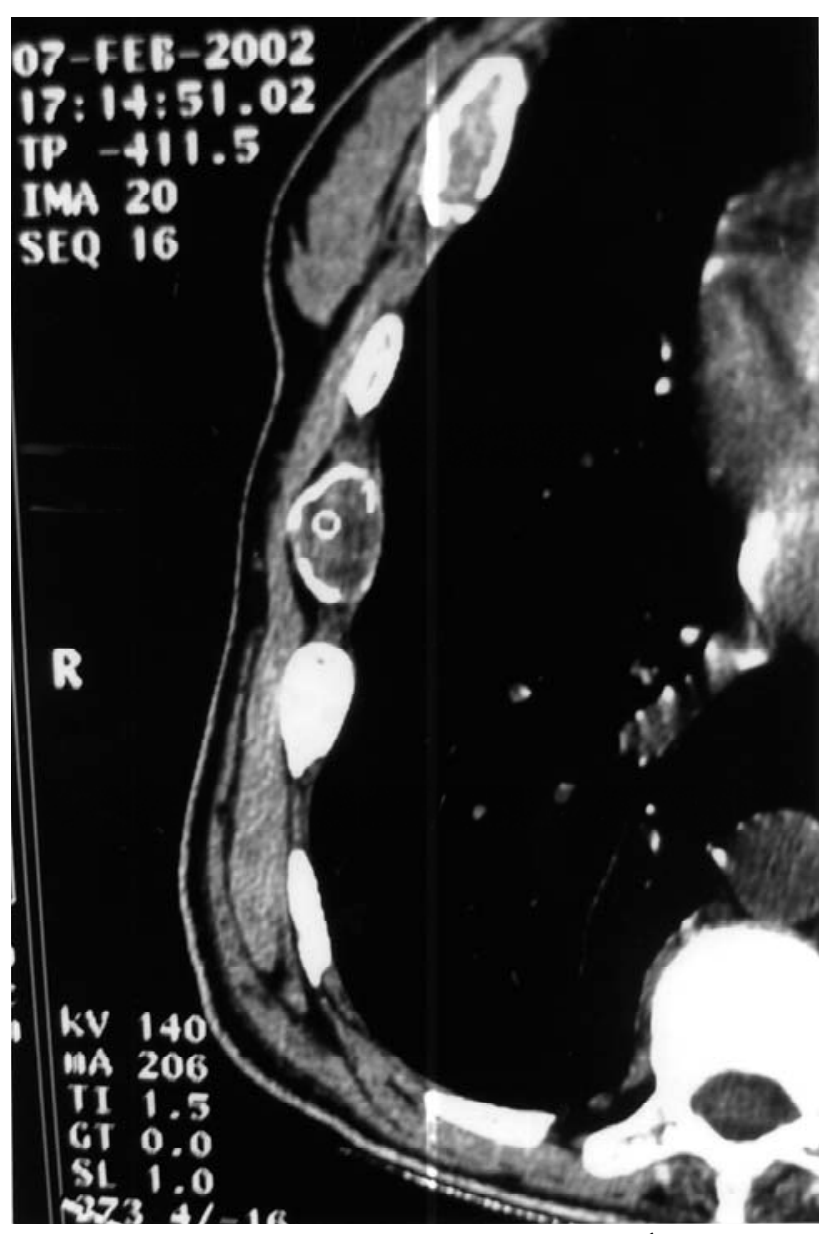

Figura 1. Tomografia computadorizada de tórax evidenciando lesões "insufladas" em arcos costais, compatíveis com tumores marrons.

projeção de paratireóide. Foi iniciado calcitonina spray nasal 400UI/dia, que a paciente usou irregularmente por quatro meses sem resposta e, posteriormente, pamidronato endovenoso $90 \mathrm{mg} / \mathrm{semana}$ por 4 semanas e hidratação venosa. Foi submetida a paratireoidectomia subtotal, e o estudo anátomo-patológico evidenciou adenoma de paratireóide. No momento encontra-se assintomática com reversão completa do quadro clínico e níveis laboratoriais pós-operatórios de PTH: $50 \mathrm{pg} / \mathrm{mL}$, FA: $150 \mathrm{U} / \mathrm{L}$, Ca: $8,5 \mathrm{mg} / \mathrm{dL}$, fósforo: $3 \mathrm{mg} / \mathrm{dL}$. A paciente não apresentou a síndrome de fome óssea no pós-operatório imediato e vem, atualmente, em uso de cálcio, vitamina $\mathrm{D}$ e prednisona $5 \mathrm{mg} /$ dia.

\section{DISCUSSĀO}

O hiperparatireoidismo é um distúrbio metabólico que resulta de uma produção anormal de paratormônio, geralmente decorrente da presença de um adenoma de 
paratireóide. Pode ocorrer em qualquer idade, mas a grande maioria dos casos ocorre em torno dos 45 anos. Mulheres são acometidas duas a três vezes mais que os homens (11).

As manifestações reumatológicas associadas ao hiperparatireoidismo primário podem ocorrer pelos seguintes mecanismos: deposição de cristais de pirofosfato de cálcio secundário à hipercalcemia (pseudo gota), hiperuricemia de causa desconhecida levando a um quadro de gota, calcificação periarticular manifestando-se como periartrite, ou, raramente, numa forma que mimetiza a artrite reumatóide e leva a deformidades mutilantes tipo "opera glass" (12). Pacientes com hiperparatireoidismo freqüentemente referem dor óssea difusa, sem evidente inflamação articular, o que certamente retarda seu diagnóstico, uma vez que essa condição muitas vezes não é lembrada. A associação de hiperparatireoidismo primário com LES é uma situação bastante rara, sendo descrita previamente em apenas uma paciente (10). As duas pacientes, a da literatura e a nossa, encontravam-se na mesma faixa etária (40-50 anos) quando tiveram diagnóstico de hiperparatireoidismo primário, com quadro clínico e laboratorial parecidos, exceto pelo tempo de diagnóstico do LES, que na nossa paciente foi de dois anos e na paciente da literatura, de dez anos; além disso, o comprometimento renal e a presença da síndrome antifosfolipídica não foram observados na nossa paciente.

A raridade dessa situação associada à falta de evidências na literatura que dêem suporte para a possibilidade de mecanismo auto-imune na etiologia do adenoma de paratireóide levantam a possibilidade dessa associação significar apenas uma coincidência de doenças. Por outro lado, pacientes com LES podem apresentar hipercalcemia, mais comumente observada devido ao hiperparatireoidismo secundário ou terciário devido à insuficiência renal crônica, por nefrite lúpica. No presente caso, a paciente apresentava função renal normal, sem qualquer comprometimento deste órgão pela doença. A hipercalcemia pode também fazer parte da "síndrome hipercalcemia-linfedema", que inclui a linfadenopatia e a pleurite $(8,9)$, manifestações estas também ausentes na nossa paciente. Em outros casos, a hipercalcemia pode ser decorrente do aumento do peptídeo relacionado à paratireóide (PTHrP), que, embora seja um conhecido mediador humoral da hipercalcemia na neoplasia (13), tem também sido relatado em condições não malignas como LES e hiperplasia mamária $(14,15)$. Adicionalmente, anticorpos contra receptores, presentes em doenças autoimunes, podem mimetizar a ação de vários peptídeos hormonais a partir da ativação de tais receptores. Sabe- se que isso pode ocorrer com os receptores do PTH levando à hipercalcemia em LES (6). Embora todas essas possibilidades possam ser aventadas, a nossa paciente apresentava altos níveis de PTH e achados cintilográficos indicando hiperfunção e aumento de paratireóide, com exame anátomo-patológico evidenciando adenoma de paratireóide.

Em conclusão, a presente experiência faz sugerir que, frente a um paciente com LES e níveis séricos elevados de cálcio, assim como em outras situações, a presença de hiperparatireoidismo primário deve ser pesquisada, uma vez que a terapêutica cirúrgica leva a uma completa resolução da hipercalcemia.

\section{REFERÊNCIAS}

1. Heath H 3rd, Hodgson SF, Kennedy MA. Primary hyperparathyroidism. Incidence, morbidity, and economic impact in a community. N Engl J Med 1982,24;302:18993.

2. Helliwell M. Rheumatic symptoms in primary hyperparathyroidism. Postgrad Med J 1983;59:236-40.

3. Bilezikian JP, Connor TB, Aptekar R, Freijanes J, Aurbach GD, Pachas WN, et al. Pseudogout after parathyroidectomy. Lancet 1973; 1:445-6.

4. Rynes RI, Merzig EG. Calcium pyrophosphate crystal deposition disease and hyperparathyroidism: a controlled, prospective study. J Rheumatol 1978;5:460-8.

5. Alexander GM, Dieppe PA, Doherty M, Scott DG. Pyrophosphate arthropathy: a study of metabolic associations and laboratory data. Ann Rheum Dis 1982;41:377-81.

6. Gazzaruso C, Montecucco CM, Geroldi D, Garzaniti A, Finardi $G$. Severe hypercalcemia and systemic lupus erythematosus. Joint Bone Spine 2000;67:485-8.

7. Deftos LJ, Burton DW, Baird SM, Terkeltaub RA. Hypercalcemia and systemic lupus erythematosus. Arthritis Rheum 1996;39:2066-9.

8. Berar-Yanay N, Weiner P, Magadle R. Hypercalcaemia in systemic lupus erythematosus. Clin Rheumatol 2001;20:147-9.

9. Katchan B, Karaplis AC, Begin LR, Trifiro M. Hypercalcaemia with lymphoedema. Lancet 1996;347:161.

10. Benekli M, Savas MC, Erdem Y, Yalcin AU, Yasavul U, Turgan $C$, et al. Primary hyperparathyroidism in a patient with systemic lupus erythematosus-antiphospholipid syndrome. Nephron 1998;79:215-6.

11. Wermers RA, Khosla S, Atkinson EJ, Grant CS, Hodgson SF, O'Fallon WM, et al. Survival after the diagnosis of hyperparathyroidism: a population-based study. Am J Med 1998; 104:115-22.

12. Bywaters EG, Dixon AS, Scott JT. Joint lesions of hyperparathyroidism. Ann Rheum Dis 1963;22:171-87.

13. Rankin W, Grill V, Martin TJ. Parathyroid hormone-related protein and hypercalcemia. Cancer 1997;80(suppl. 


\section{8):1564-71.}

14. Khosla S, van Heerden JA, Gharib H, Jackson IT, Danks J, Hayman JA, et al. Parathyroid hormone-related protein and hypercalcemia secondary to massive mammary hyperplasia. N Engl J Med 1990;322:1157.

15. Knecht TP, Behling CA, Burton DW, Glass CK, Deftos LJ. The humoral hypercalcemia of benignancy. A newly appre- ciated syndrome. Am J Clin Pathol 1996; 105:487-92. Endereço para correspondência:

Mittermayer B. Santiago

Núcleo de Reumatologia da Bahia

Hospital Santa Izabel

Praça Almeida Couto 500

40000-000 Salvador, BA

E-mail: mitter@svn.com.br 